\title{
DETECÇÃO DE ENCEFALOPATIA HEPÁTICA MÍNIMA ATRAVÉS DE TESTES NEUROPSICOLÓGICOS E NEUROFISIOLÓGICOS E O PAPEL DA AMÔNIA NO SEU DIAGNÓSTICO
}

\author{
Maurício Augusto BRAGAGNOLO Jr. ${ }^{1}$, Vinícius TEODORO², Lígia Mendonça LUCCHESI ${ }^{2}$, \\ Tarsila Campanha da Rocha RIBEIRO' ${ }^{1}$, Sérgio TUFIK² e Mário KONDO1
}

\begin{abstract}
RESUMO - Contexto - A encefalopatia hepática mínima vem sendo sistematicamente investigada em pacientes com cirrose hepática. Entretanto, existem controvérsias quanto aos melhores métodos, bem como o papel da amônia para seu diagnóstico. Objetivo - Avaliar a frequência de encefalopatia hepática mínima diagnosticada através de testes neuropsicológicos e neurofisiológicos em cirróticos, bem como os possíveis fatores de risco para esta condição, incluindo o papel da concentração arterial de amônia em seu diagnóstico. Métodos - Indivíduos com cirrose hepática foram avaliados através do teste de conexão numérica partes A e B (TCN-A e TCN-B) e potencial evocado relacionado a eventos (P300). O diagnóstico de encefalopatia hepática mínima foi feito quando da presença de anormalidade no P300 e em, pelo menos, um dos testes neuropsicológicos. As concentrações arteriais de amônia, a escolaridade e a gravidade da cirrose hepática também foram avaliadas em todos. Resultados - Foram avaliados 48 pacientes cirróticos, com média de idade $50 \pm 8$ anos, sendo $79 \%$ do sexo masculino. As principais causas foram a alcoólica e a viral. O P300 foi anormal em 75\% dos casos e o TCN-A e TCN-B anormais em 58\% e $65 \%$ dos casos, respectivamente. Os resultados do TCN-B foram influenciados pela escolaridade. A frequência de encefalopatia hepática mínima foi de 50\%. A concentração arterial de amônia não foi significantemente maior em pacientes com diagnóstico de encefalopatia hepática mínima (195 $\pm 152 \mathrm{mmol} / \mathrm{L}$ versus $148 \pm 146 \mathrm{mmol} / \mathrm{L} ; P>0,05)$. Não houve diferença significante entre os grupos com e sem encefalopatia hepática mínima quanto às demais variáveis estudadas. Conclusão - A encefalopatia hepática mínima é condição frequente em pacientes com cirrose hepática. A concentração arterial de amônia não parece ter papel importante no seu diagnóstico.
\end{abstract}

DESCRITORES - Encefalopatia hepática, diagnóstico. Cirrose hepática. Potencial evocado P300. Testes neuropsicológicos. Amônia.

\section{INTRODUÇÃO}

A encefalopatia hepática (EH) pode ser conceituada como um conjunto de alterações neuropsiquiátricas que ocorrem em indivíduos com insuficiência hepática e/ou hipertensão portal, na ausência de distúrbios metabólicos e/ou neuropsiquiátricos não relacionados ${ }^{(7)}$. Sua importância prognóstica é reconhecida, já que se estima em $42 \%$ a sobrevida em 1 ano para indivíduos com cirrose após o desenvolvimento desta complicação $0^{(3)}$. Sua fisiopatologia é complexa e possivelmente multifatorial, entretanto a amônia ainda parece ser a candidata que melhor explica as alterações clínicas e neuropatológicas observadas na $\mathrm{EH}^{(4)}$

Nas últimas décadas vem sendo descrita a entidade denominada encefalopatia hepática mínima (EHM). O termo se aplica àqueles pacientes com insuficiência hepática e/ou hipertensão portal que não apresentam alterações neuropsiquiátricas perceptíveis ao exame clínico, mas que manifestam alterações detectáveis em testes neuropsicológicos e/ou neurofisiológicos apropriados ${ }^{(7)}$. A EHM é, portanto, uma condição não percebida quando do exame clínico e, durante vários anos, sua presença foi considerada, por este motivo, irrelevante. Entretanto, estudos clínicos demonstram que cirróticos com EHM apresentam pior qualidade de vida $^{(8)}$, prejuízo da memória e da atenção ${ }^{(36)}$ e dificuldade para realizar atividades cotidianas simples como, por exemplo, dirigir veículos automotivos ${ }^{(33)}$. Da mesma forma, a EHM vem sendo considerada estágio pré-clínico da $\mathrm{EH}$, desde que sua evolução para $\mathrm{EH}$ clinicamente manifesta se faz em cerca de $50 \%$ dos casos em curtos períodos de seguimento ${ }^{(28,29)}$.

Instituição: Universidade Federal de São Paulo / Escola Paulista de Medicina (UNIFESP/EPM), São Paulo, SP.

Departamentos de ${ }^{1}$ Gastroenterologia e de ${ }^{2}$ Psicobiologia UNIFESP/EPM.

Endereço: Dr. Maurício A. Bragagnolo Jr. - Rua Clóvis Serôa da Motta, 138 - Bairro São Geraldo - 36031-280 - Juiz de Fora, MG. E-mail: mauricio.jr@acessa.com 
A forma de se diagnosticar a EHM, no entanto, é ainda controversa. Um bom exemplo deste fato é a grande variação na sua prevalência (23\% a 84\%), conforme o grupo e a metodologia utilizada para sua detecção ${ }^{(9,18,26,30,34)}$. Segundo as recomendações consensuais $^{(7)}$, devem ser utilizados pelo menos dois testes neuropsicológicos e, quando possível, testes neurofisiológicos, como o eletroencefalograma e o potencial evocado relacionado a eventos (P300).

No Brasil, poucos estudos existem no sentido de determinar a prevalência de EHM em pacientes com cirrose hepática $(\mathrm{CH})$ e também pouco se conhece sobre os fatores envolvidos em sua gênese, tais como o sexo, a idade, a raça, a gravidade da $\mathrm{CH}$, suas diferentes causas, entre outras variáveis ${ }^{(5)}$. Embora a amônia seja a principal substância envolvida na fisiopatologia da $\mathrm{EH}^{(19)}$, pouco se conhece sobre o seu papel no diagnóstico da EHM. Além do mais, frequentemente lida-se com indivíduos com baixa escolaridade, o que poderia ser problema para o diagnóstico desta entidade, desde que alguns testes neuropsicológicos podem ser influenciados por esta variável ${ }^{(17,38)}$. A utilização de testes neurofisiológicos mais sofisticados, que poderiam minimizar a interferência da escolaridade sobre os resultados dos exames, como o P300, ainda é bastante limitada pela relativa indisponibilidade deste método em diversos serviços de hepatologia.

\section{Objetivos}

Avaliar a frequência de EHM em indivíduos com $\mathrm{CH}$ e os possíveis fatores envolvidos no seu diagnóstico, inclusive as concentrações arteriais de amônia.

\section{MÉTODOS}

\section{Avaliação clínica e laboratorial}

Foram avaliados pacientes com diagnóstico de $\mathrm{CH}$, provenientes do ambulatório de hepatologia da Universidade Federal de São Paulo / Escola Paulista de Medicina, São Paulo, SP, no período de março de 2002 a março de 2004. A avaliação inicial incluiu anamnese detalhada, exame físico geral, exame neuropsiquiátrico e avaliação laboratorial. Foram excluídos do estudo aqueles com EH clinicamente manifesta, os que apresentaram sangramento digestivo no último mês, indivíduos com insuficiência renal (definida como creatinina sérica $\geq 2,0 \mathrm{mg} / \mathrm{dL}$ ), diagnóstico de $\mathrm{CH}$, uso de medicações psicoativas ou lactulose nas últimas 2 semanas, uso de álcool nos últimos 6 meses, idade inferior a 18 anos ou superior a 65 anos e analfabetismo.

Todos foram submetidos a punção venosa periférica para avaliação das provas hepáticas (bilirrubinas, tempo e atividade de protrombina, albumina) e creatinina sérica, além de punção arterial para dosagem de amônia (método azul de bromocresol; valor de referência = 9-33 mmol/L). O estádio da doença hepática foi feito através da classificação de Child modificada por Pugh, sendo considerados Child A aqueles com 5-6 pontos, Child B aqueles com 7-9 e Child $\mathrm{C}$ aqueles com 10 ou mais pontos ${ }^{(25)}$.

Foi determinada a escolaridade de cada indivíduo através de questionamento direto. Os pacientes foram subdivididos em 2 grupos: grupo X - indivíduos com escolaridade muito baixa ou baixa (até primário incompleto e até ginasial incompleto, respectivamente) e grupo $\mathrm{Y}$ - indivíduos com escolaridade razoável, boa e muito boa (até colegial incompleto, superior incompleto e superior completo, respectivamente).

Após explicação detalhada do projeto, dos riscos e benefícios dos exames a serem empregados, os pacientes que concordaram em participar do estudo assinaram um termo de consentimento livre e informado, aprovado pela Comissão de Ética Médica da instituição.

\section{Avaliação neuropsicológica}

A avaliação neuropsicológica foi feita através da realização dos testes de conexão numérica parte A (TCN-A) e B (TCN-B), sempre pelo mesmo examinador, em uma mesma sala, utilizando apenas papel e lápis. Para o TCN-A a tarefa foi conectar números em ordem crescente $(1,2,3 \ldots 25)$, no menor tempo possível. Para o TCN-B, conectar números, em ordem crescente, as letras em ordem alfabética, de forma alternada (1-A, 2-B, 3-C e assim por diante). O tempo foi cronometrado e o resultado dos exames foi expresso pelo tempo em segundos, utilizado para a realização das tarefas. Aqueles que ultrapassaram o tempo de 5 minutos para sua realização tiveram como resultado o valor de 300 segundos. Foram considerados anormais os testes cujo tempo para realização do exame ultrapassasse a média $\pm 2 \mathrm{DP}$ do considerado normal para a faixa etária, segundo padronização destes testes pela literatura ${ }^{(35)}$.

\section{Avaliação neurofisiológica}

A avaliação neurofisiológica foi feita através do ERP-P300, sendo utilizado aparelho de marca Nihon Konden, Neuropack, MEB5508K. O exame foi realizado sempre por um mesmo examinador, num mesmo ambiente, com iluminação e temperatura padronizadas. Eletrodos ativos foram colocados em FZ, CZ e PZ, de acordo com o sistema internacional $10-20^{(12)}$. A impedância elétrica foi mantida abaixo de $5 \mathrm{~K} \Omega$. Os estímulos auditivos foram apresentados bilateralmente através de fones de ouvido com tons de $70 \mathrm{db}$. Dois tons foram apresentados de forma aleatória: estímulo raro, de $1500 \mathrm{~Hz}$ (com 20\% de probabilidade de aparecimento) e estímulo frequente, de $800 \mathrm{~Hz}$ (com $80 \%$ de probabilidade). O paradigma utilizado foi o "oddball"(24). Portanto, solicitou-se ao examinando que apertasse um botão toda vez que escutasse um som raro, com a mão dominante, tão rápida e acuradamente quanto possível. A latência da onda P300 foi dada pela medida da latência entre o estímulo dado e o surgimento da primeira e maior onda positiva registrada entre 250 e $500 \mathrm{~ms}$ do estímulo raro.

Para estimar o limite superior da normalidade para a latência da onda P300 aferida em Cz para determinada idade, foi utilizada a fórmula $y=(2,23 x$ idade $)+234$, conforme estudo normativo previamente realizado em nosso meio ${ }^{(16)}$. O P300 foi considerado anormal quando a latência da onda P300 era maior que o limite superior da normalidade calculado pela fórmula.

\section{Diagnóstico de EHM}

O diagnóstico de EHM foi firmado quando da presença de anormalidade no P300 e em, pelo menos, um dos testes neuropsicológicos realizados, segundo as recomendações consensuais proferidas por FERENCI et al. ${ }^{(7)}$, em 2002. 


\section{Análise estatística}

A análise estatística foi feita utilizando-se o programa Statistica, versão 5.0 (edição 97). As comparações entre grupos das variáveis quantitativas deste estudo foram realizadas através do teste $t$ de Student, enquanto que as comparações entre grupos das variáveis qualitativas foram realizadas através do teste qui ao quadrado. A correlação de Spearman foi utilizada quando indicada. O nível de significância adotado foi o de 5\%.

\section{RESULTADOS}

No total, foram avaliados 48 pacientes com $\mathrm{CH}$, com média de idade de $50 \pm 8$ anos, sendo 38 (79\%) do sexo masculino. As causas mais frequentes foram: cirrose alcoólica em 21 pacientes (44\%) e cirrose por vírus da hepatite C em 12 pacientes $(25 \%)$. Quanto à gravidade da doença, 21 (44\%) pacientes eram Child A, 18 (37\%) Child B e 9 (19\%) Child C, com média de 7,3 \pm 2,1 pontos. Quanto à escolaridade, 26 pertenciam ao grupo $\mathrm{X}$ e 22 ao grupo Y (22 pacientes). O número médio de anos de escolaridade foi de $6,7 \pm 2,8$ (1-11 anos de escolaridade). As características gerais do grupo estudado são resumidas na Tabela 1 .

TABELA 1. Características gerais do grupo estudado

\begin{tabular}{lc}
\hline \multicolumn{1}{c}{ Características } & Média \pm DP ou número (\%) \\
\hline Idade & $50 \pm 8$ anos \\
Sexo masculino & $38(79 \%)$ \\
Causa & \\
$\quad$ Álcool & $21(44 \%)$ \\
$\quad$ Vírus da hepatite C & $12(25 \%)$ \\
$\quad$ Álcool + vírus da hepatite C & $8(17 \%)$ \\
$\quad$ Vírus da hepatite B & $2(04 \%)$ \\
Álcool + vírus da hepatite B & $1(02 \%)$ \\
$\quad$ Criptogênica & $4(08 \%)$ \\
Anos escolaridade & $6,7 \pm 2,8$ \\
Escolaridade & \\
$\quad$ grupo X* & \\
$\quad$ grupo Y** & $26(54 \%)$ \\
Pontos Child-Pugh & $22(46 \%)$ \\
Child A & $7,3 \pm 2,1$ \\
Child B & $21(44 \%)$ \\
Child C & $18(37 \%)$ \\
\hline
\end{tabular}

* grupo $X:$ indivíduos com escolaridade muito baixa ou baix

** grupo $Y$ : indivíduos com escolaridade razoável, boa e muito boa

O tempo médio para a realização do TCN-A foi de $74 \pm$ 56 segundos, anormal em 28 (58\%) pacientes. O tempo médio para a realização do TCN-B foi de $196 \pm 84$ segundos, anormal em 31 (65\%) pacientes. Em relação ao P300, observou-se latência média da onda P300 de $365,4 \pm 42,16 \mathrm{~ms}$ para o grupo (282 a $496 \mathrm{~ms})$. Trinta e seis pacientes $(75 \%)$ obtiveram retardo da latência, ou seja, P300 anormal. Observou-se correlação positiva de moderada a forte, entre o tempo para realização do TCN-A e do TCN-B (Correlação de Spearman, $\mathrm{R}=+0,73, P<0,01$ ) Figura 1. No entanto, quando se avaliou a correlação entre a latência da onda P300 e o tempo para a realização do TCN-A e do TCN-B, foi observada apenas fraca correlação estatística (correlação de Spearman, $\mathrm{R}=+0,30 ; P=0,04$ e $\mathrm{R}=+0,29 ; P>0,05$, respectivamente) Figuras 2 e 3.

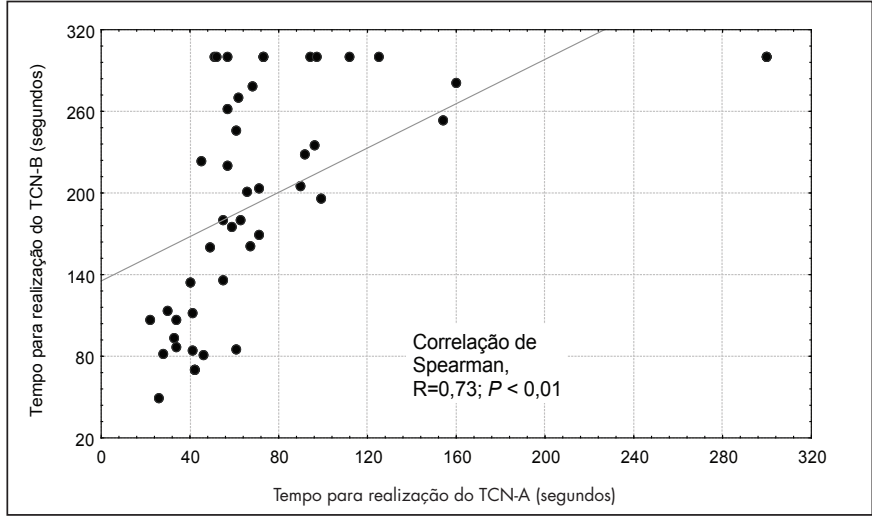

FIGURA 1. Correlação entre o tempo para realização dos testes neuropsicológicos TCN-A e TCN-B

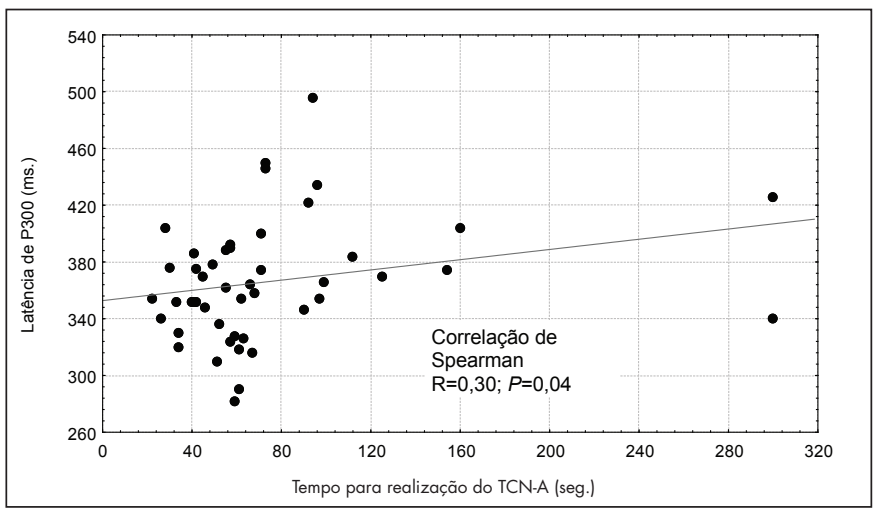

FIGURA 2. Correlação entre o tempo para realização do ERP-P300 e do TCN-A

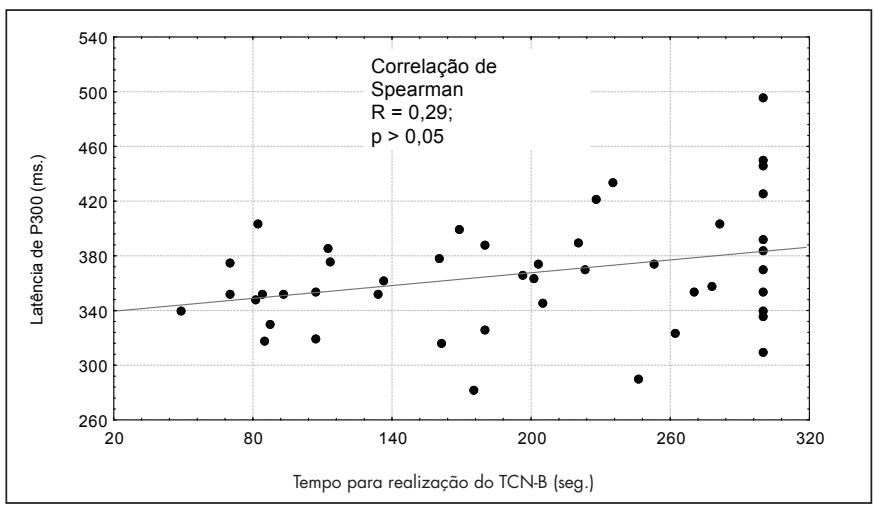

FIGURA 3. Correlação entre o tempo para realização do ERP-P300 e do TCN-B

O grau de escolaridade não influenciou os valores da latência da onda P300 (grupo $\mathrm{X}=372 \pm 76 \mathrm{~ms}$ versus grupo $\mathrm{Y}=358 \pm$ $36 \mathrm{~ms} ; P>0,05$ - teste $t$ de Student). $\mathrm{O}$ tempo para realização do TCN-A em indivíduos dos grupos X e Y também não foi estatisticamente diferente $(80 \pm 70$ versus $68 \pm 37$ segundos, respectivamente; $P>0,05$ - teste $t$ de Student). O mesmo, 
entretanto, não ocorreu com o TCN-B, sendo que o tempo para realização deste teste foi significantemente maior no grupo com pior escolaridade em relação ao grupo com melhor escolaridade (grupo X: $223 \pm 79$ versus grupo Y: $167 \pm 82$ segundos; $P<0,05$ - teste $t$ de Student) Figura 4.

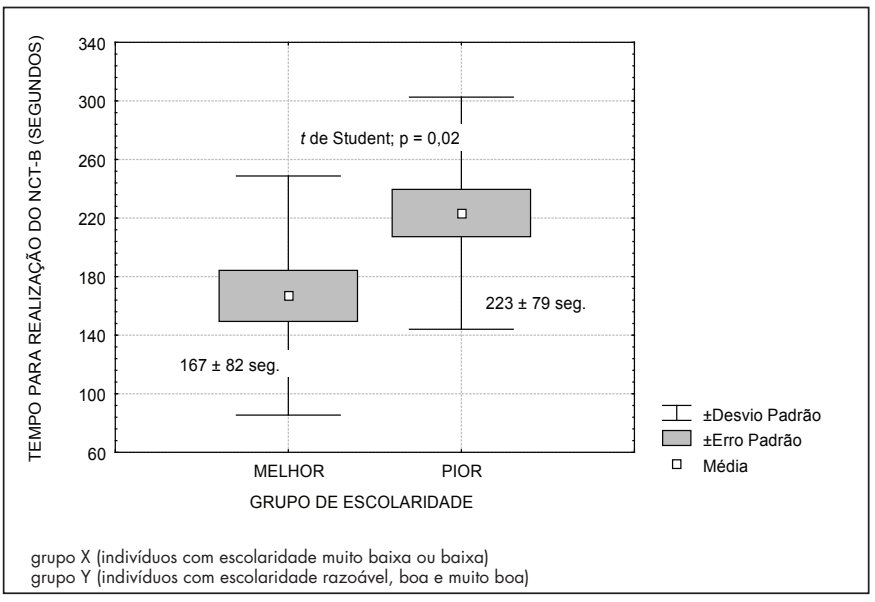

FIGURA 4. Comparação do tempo para realização do TCN-B nos diferentes grupos de escolaridade

A frequência de EHM no grupo estudado, segundo a metodologia adotada, foi de 50\%. O diagnóstico de EHM foi dado por alteração no $\mathrm{P} 300+\mathrm{TCN}-\mathrm{A}$ e TCN-B em 20 pacientes e por alteração no P300 + TCN-B em 4 pacientes. No entanto, todos os pacientes com diagnóstico de EH firmado pelo último método pertenciam ao grupo com escolaridade mais baixa, isto é, o grupo X. Nenhum paciente teve diagnóstico de EHM por alteração no P300 + TCN-A, exclusivamente.

Os grupos com e sem EHM foram homogêneos quanto ao sexo, idade, raça, grupos e anos de escolaridade. A frequência desta condição não foi significantemente diferente nas diferentes causas de $\mathrm{CH}$. A gravidade da $\mathrm{CH}$ avaliada pela classificação de Child-Pugh, também não esteve associada a maior frequência EHM e o número médio de pontos obtidos na classificação também não foi significantemente diferente em pacientes com e sem EHM (7,4 $\pm 2,0$ versus 7,3 $\pm 2,2 ; P>0,05$ - teste $t$ de Student) Tabela 2.

A dosagem de amônia foi realizada em 43 pacientes, sendo a média de $172 \pm 149 \mathrm{mmol} / \mathrm{L}(33-543 \mathrm{mmol} / \mathrm{L})$. Apenas um paciente apresentou concentração arterial de amônia normal, isto é, a prevalência de hiperamonemia em cirróticos foi de $98 \%$. Os níveis séricos de amônia não foram significantemente diferentes em cirróticos com e sem EHM $(195 \pm 152 \mathrm{mmol} / \mathrm{L}$ versus. $148 \pm 146 \mathrm{mmol} / \mathrm{L} ; P>0,05)$ Figura 5.

\section{DISCUSSÃo}

A CH é uma doença frequente em nosso meio e é associada à significante morbidade e mortalidade. No Brasil, dados do Ministério da Saúde revelam que são notificados anualmente 8.870 óbitos/ano, secundários à cirrose e suas complicações, entre elas a EH ${ }^{(2)}$. Embora a importância da EHM seja reconhecida, seu
TABELA 2. Comparação entre indivíduos com cirrose hepática com e sem encefalopatia hepática mínima

\begin{tabular}{|c|c|c|c|}
\hline Características & $\begin{array}{c}\text { Sem EHM } \\
(\mathrm{n}=24)\end{array}$ & $\begin{array}{c}\text { Com EHM } \\
(\mathrm{n}=24)\end{array}$ & $P$ \\
\hline Sexo masculino & 20 & 18 & 0,47 \\
\hline Idade (anos) & $49 \pm 7$ & $51 \pm 10$ & 0,48 \\
\hline Raça branca & 21 & 18 & 0,27 \\
\hline Anos escolaridade & $7,2 \pm 2,8$ & $6,2 \pm 2,8$ & 0,25 \\
\hline \multicolumn{4}{|l|}{ Escolaridade } \\
\hline Grupo $X^{*}$ & 13 & 13 & 1,0 \\
\hline Grupo $\mathrm{Y}^{* *}$ & 11 & 11 & 1,0 \\
\hline \multicolumn{4}{|l|}{ Causa da cirrose } \\
\hline Alcoólica & 10 & 11 & 0,77 \\
\hline Vírus da hepatite $C$ & 6 & 6 & 1,00 \\
\hline Álcool + vírus da hepatite C & 5 & 3 & 0,43 \\
\hline Vírus da hepatite $\mathrm{B}$ & 1 & 1 & 1,00 \\
\hline Álcool + Vírus da hepatite B & 0 & 1 & 0,31 \\
\hline Criptogênica & 2 & 2 & 1,00 \\
\hline \multicolumn{4}{|l|}{ Classe de Child-Pugh } \\
\hline A & 10 & 11 & 0,77 \\
\hline B & 10 & 8 & 0,55 \\
\hline C & 4 & 5 & 0,71 \\
\hline Pontos Child-Pugh & $7,3 \pm 2,2$ & $7,4 \pm 2,0$ & 0,89 \\
\hline
\end{tabular}

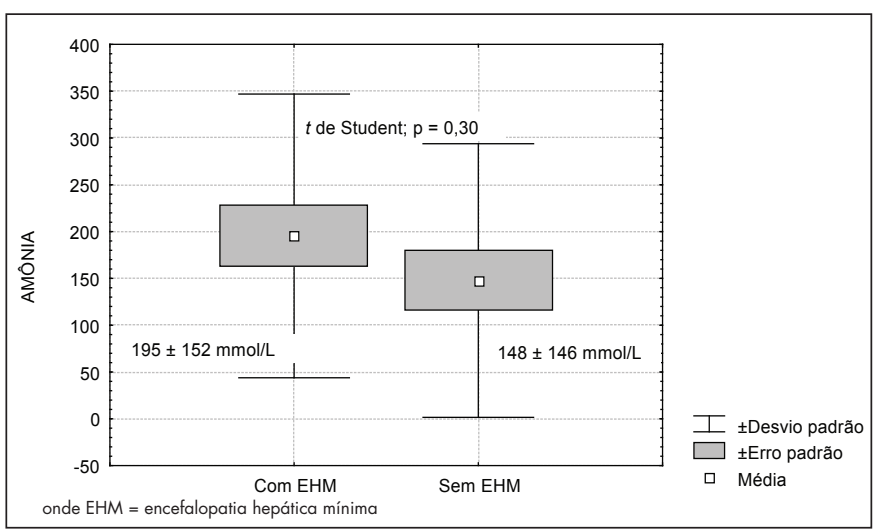

FIGURA 5. Concentração arterial de amônia em indivíduos com cirrose hepática com e sem encefalopatia hepática mínima

diagnóstico é, por outro lado, bastante controverso. Possível fosse listar todos os critérios diagnósticos já utilizados até o momento, poder-se-ia mencionar combinação quase que infindável entre diversos testes neuropsicológicos e neurofisiológicos. O presente estudo utilizou o TCN-A e o TCN-B, métodos que avaliam a habilidade cognitiva motora, sendo os mais frequentemente usados para a detecção de EHM, de forma geral ${ }^{(26)}$. Na amostra estudada, foram anormais em $58 \%$ e $65 \%$ dos casos, respectivamente, em congruência com os resultados encontrados por vários autores. Conforme descrito previamente, o resultado destes exames sofre, pelo menos em certo grau, influência da escolaridade ${ }^{(17,35,38)}$. Na presente amostra este fato pode ser observado nitidamente, pelo menos no que diz respeito aos resultados do TCN-B. Pacientes com escolaridade baixa ou muito baixa apresentaram tempo médio para a realização do teste significantemente maior, quando comparados àqueles com escolaridade regular, boa ou muito boa $(223 \pm 79$ versus $167 \pm 82$ segundos; $P<0,05)$. Cabe ainda 
ressaltar que se estima escolaridade mais baixa nos pacientes atendidos no ambulatório de hepatologia da anteriormente referida instituição, considerando a pré-seleção de pacientes a serem incluídos no estudo, isto é, a exclusão dos analfabetos.

O presente estudo segue a tendência atual de se utilizar a combinação de testes neuropsicológicos e um teste neurofisiológico. O P300 vem sendo utilizado nas últimas décadas para avaliar enfermidades que cursam com alterações cognitivas, entre elas a

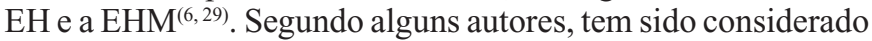
o melhor teste e o mais sensível para o diagnóstico de $\mathrm{EHM}^{(15,37)}$. Observa-se nestes casos, retardo na latência da onda P300, o que reflete disfunção cognitiva, sobretudo quanto à rapidez para processar e manter a atenção em novas informações ${ }^{(20,21)}$. Entretanto, foi utilizado previamente apenas em poucos estudos ${ }^{(3,6,10,27,29)}$. No presente trabalho, o P300 esteve alterado em 75\% dos casos. KUGLER et al. ${ }^{(14)}$ também descreveram anormalidade no resultado do $\mathrm{P} 300$ de cirróticos, em $78 \%$ dos casos. No entanto, a latência da onda P300 não correlacionou de forma significante com o tempo para a realização do TCN-A e TCN-B (correlação de Spearman com $\mathrm{R}=+0,30$ e $+0,29$, respectivamente), testes estes mais frequentemente utilizados na prática clínica ${ }^{(26)}$. Embora ainda não exista um exame padrão-ouro para o diagnóstico de EHM e o papel do P300 neste contexto ainda seja pouco conhecido, na presente casuística apresentou a vantagem de não ser influenciado pela escolaridade, quando comparado aos testes neuropsicológicos, o que parece ser interessante, sobretudo para populações heterogêneas e para aquelas com baixo grau de escolaridade. Embora haja a necessidade de equipamento específico para sua realização, também se destacou em outros estudos por não sofrer influência do aprendizado e do grau de escolaridade ${ }^{(22)}$.

A frequência de EHM no grupo estudado foi de $50 \%$, semelhante à descrita na literatura ${ }^{(9,18,26,30,35)}$. O diagnóstico de EHM foi firmado quando da alteração no P300 e em pelo menos um dos testes neuropsicológicos. A forma de diagnóstico de EHM mais frequente foi alteração no P300 associada à alteração do TCN-A e TCN-B. Apenas quatro pacientes foram diagnosticados através de alteração no P300 e no TCN-B isoladamente. Nenhum paciente com diagnóstico de EHM teve alteração do P300 e do TCN-A isoladamente, ou seja, sempre que o TCN-A esteve alterado, o TCN-B também esteve. Tais achados podem sugerir maior sensibilidade do TCN-B para o diagnóstico de EHM, quando comparado ao TCN-A, embora não sugira, necessariamente, maior especificidade. Por outro lado, considerando que o tempo para realização do TCN-B foi significantemente maior naqueles do grupo $\mathrm{X}$ (menor escolaridade) e que todos os quatro pacientes com EHM firmada por alteração do P300 + TCN-B pertencessem a este grupo, poder-se-ia também supor que o mesmo deveria, não necessariamente, ter um diagnóstico correto da afecção (falso-positivo), desde que esta constatação pudesse estar simplesmente associada à baixa escolaridade que possuía e não, realmente, à presença da EHM. Sendo assim, seriam na realidade apenas 20 pacientes com esta condição, sendo que a verdadeira frequência de EHM seria pouco mais baixa do que a assumida, ou seja, em $42 \%$ da amostra.

A presença de EHM não foi influenciada pelo sexo, idade, raça e etiologia da doença hepática. Sua prevalência também não foi maior em indivíduos com insuficiência hepática mais grave (Child $\mathrm{A}=52 \%$, Child $\mathrm{B}=44 \%$, Child $\mathrm{C}=55 \%$ ). Alguns autores já descreveram menor prevalência de EHM em indivíduos Child A, quando comparados aos Child B e C (14\% vs 44\%; $P<0,05)^{(26)}$, enquanto outros não observaram o mesmo achado ${ }^{(33)}$. As diferentes amostras e as variações metodológicas utilizadas nos diversos estudos talvez possam explicar as discrepâncias encontradas.

A prevalência de hiperamonemia na amostra estudada, ou seja, cirróticos sem EH, foi de $98 \%$, com grande variabilidade nos níveis arteriais de amônia observados (amplitude da amostra de $510 \mathrm{mmol} / \mathrm{L}$ ). De forma semelhante, um estudo clássico ${ }^{(31)}$ também já mostrou grande variação nos níveis de amônia e no teste de tolerância à amônia em cirróticos sem EH $(97,6 \%$ dos casos). Estes achados, reproduzidos posteriormente ${ }^{(17,23)}$, levam a permanente descrédito no que diz respeito à importância da hiperamonemia para o diagnóstico de $\mathrm{EH}$, desde que pacientes com cirrose, mesmo sem esta condição clínica, quase que invariavelmente apresentam elevações séricas desta substância. No presente estudo, a presença de hiperamonemia também não auxiliou no diagnóstico da $\mathrm{EH}$ em sua fase mais inicial, isto é, a EHM, desde que não houve diferença estatisticamente significante entre as concentrações arteriais de amônia em indivíduos com e sem esta condição $(195 \pm 152 \mathrm{mmol} / \mathrm{L}$ versus $148 \pm 146 \mathrm{mmol} / \mathrm{L}$; $P>0,05)$. Esta observação, no entanto, ainda é digna de reprodução, desde que pouco se conhece sobre esta relação.

\section{CONCLUSÕES}

A EHM é uma condição comumente encontrada em cirróticos e sua frequência foi de 50\%, quando diagnosticada através de testes neuropsicológicos e potencial evocado relacionado a eventos. Embora seja necessária maior investigação sobre o verdadeiro papel do P300 no diagnóstico da EHM, este se mostrou como ferramenta interessante, uma vez que não sofreu influência da escolaridade, ao contrário dos testes neuropsicológicos. No grupo estudado, a concentração arterial de amônia não se correlacionou com o diagnóstico de EHM. 
Bragagnolo Jr MA, Teodoro V, Lucchesi LM, Ribeiro TCR, Tufik S, Kondo M. Minimal hepatic encephalopathy detection by neuropsychological and neurophysiological methods and the role of ammonia for its diagnosis. Arq Gastroenterol. 2009;46(2): 43-9.

ABSTRACT - Context - Minimal hepatic encephalopathy has been systematically investigated in cirrhotic patients. Although, there are controversies regarding the best methods as well as the role of ammonia for its diagnosis. Objective - To evaluate the frequency of minimal hepatic encephalopathy diagnosed by neuropsychological and neurophysiological methods in cirrhotic patients, as well as possible associated risk factors for this condition, including the role of arterial ammonia concentrations for its diagnosis. Methods - Cirrhotic patients were evaluated by the number connection test parts A and B (NCT-A and NCT-B), and auditory evoked-related potentials (P300). Minimal hepatic encephalopathy was diagnosed by the presence of abnormal P300 and in unless one of the performed neuropsychologic tests. Arterial ammonia concentration, scholarity and cirrhosis severity accessed by Child-Pugh classification were evaluated in all. Results - Forty-eight cirrhotic patients were evaluated, with median age $50 \pm 8$ years old, $79 \%$ male. The main etiologies were alcoholic and viral. The P300 was abnormal in 75\% of cases, while NCT-A and NCT-B were abnormal in 58\% and $65 \%$, respectively. The NCT-B results were influenced by scholarity. The minimal hepatic encephalopathy frequency was $50 \%$. Arterial ammonia concentration was not significantly increased in minimal hepatic encephalopathy diagnosed patients $(195 \pm 152 \mathrm{mmol} / \mathrm{L}$ versus $148 \pm 146 \mathrm{mmol} / \mathrm{L} ; P>0,05)$. There was no difference between groups with or without minimal hepatic encephalopathy in the other studied variables. Conclusion - Minimal hepatic encephalopathy is a frequent condition in cirrhotic patients. The arterial ammonia concentration does not play a major role in its diagnosis.

HEADINGS - Hepatic encephalopathy, diagnosis. Liver cirrhosis. Event-related potentials, P300. Neuropsychological tests. Ammonia.

\section{REFERÊNCIAS}

1. Bessman SP, Bradley JE. Uptake of ammonia by muscle. N Engl J Med. 1955;253:1143-7.

2. Brasil. Ministério da Saúde. C.16 Taxa de mortalidade específica por cirrose hepática. http://www.datasus.tabnet.gov.br.

3. Bustamante J, Rimola A, Ventura PJ, Navassa M, Cirera I, Reggiardo V, Rodes J. Prognostic significance of hepatic encephalopathy in patients with cirrhosis. J Hepatol. 1999;30:890-5.

4. Butterworth RF. Pathophysiology of hepatic encephalopathy: a new look at ammonia. Metab Brain Dis. 2002; 17:221-7.

5. Dantas-Corrêa EB. Avaliação neuropsicológica da atenção em pacientes portadores de cirrose hepática não alcoólica na ausência de encefalopatia hepática [tese]. São Paulo: Universidade Federal de São Paulo, Escola Paulista de Medicina; 2003.

6. Davies MG, Rowan MJ, Macmathuna P, Keeling PWN, Weir DG, Feely J. The auditory P300 event-related potential: an objective marker of the encephalopathy of chronic liver disease. Hepatology. 1990;12:688-94.

7. Ferenci P, Lockwood A, Mullen K, Tarter R, Weissenborn K, Blei AT. Hepatic encephalopathy - definition, nomenclature, diagnosis, and quantification: final report of the Working Party at the 11th World Congresses of Gastroenterology, Vienna, 1998 Hepatology. 2002;35:716-21.

8. Groeneweg M, Quero JC, De Bruijn I, Hartmann IJC, Essink-Bot ML, Hop WCJ, Schalm SW. Subclinical hepatic encephalopathy impairs daily functioning. Hepatology. 1998;28:45-9.

9. Groeneweg M, Moerland W, Quero JC, Hop WCJ, Krabbe PF, Schalm SW. Screening of subclinical hepatic encephalopathy. J Hepatol. 2000;32:748-53.

10. Hollerbach S, Kullmann F, Rudiger F, Lock G, Geissler A, Scholmerich J, Holstege A. Auditory event-related cerebral potentials (P300) in hepatic encephalopathy topographic distribution and correlation with clinical and psychometric assessment. Hepatogastroenterology. 1997;44:1002-12.

11. Instituto Brasileiro de Geografia e Estatística - IBGE. Síntese de indicadores sociais 2002, n. 11, 2003.

12. Jasper HH. Report of the committee on methods of clinical examination in electroencephalography. Electroencephalogr Clin Neurophisiol. 1958;10:370-5.

13. Kramer L, Tribl B, Gendo A, Zauner C, Schneider B, Ferenci P, Madl C. Partial pressure of ammonia versus ammonia in hepatic encephalopathy. Hepatology. 2000;31:30.

14. Kugler CFA, Lotterer E, Petter J, Wensing G, Taghavy A, Hahn EG, Fleig WE. Visual event-related $\mathrm{P} 300$ potentials in early portosystemic encephalopathy. Gastroenterology. 1992;103:302-10.

15. Kullmann F, Hollerbach S, Holstege A, Scholmerich J. Subclinical hepatic encephalopathy: the diagnostic value of evoked potentials. J Hepatol. 1995;22:101-10.

16. Luccas FJC, Lucchesi LM, Lopes JA. Potenciais evocados ligados a eventos P300: estudo normativo. (Apresentado no $13^{\circ}$ Congresso Brasileiro de Neurologia; 1988; São Paulo, SP. Anais do Congresso. p. 33).
17. Marchesini G, Zoli M, Dondi C, Cecchini L, Angiolini A, Bianchi FB, Pisi E. Prevalence of subclinical hepatic encephalopathy in cirrhosis and relationship to plasma aminoacid imbalance. Dig Dis Sci. 1980;25:763-8.

18. Moore JW, Dunk AA, Crawford JR, Deans H, Besson JA, De Lacey G, Sinclair TS, Mowat NA, Brunt PW. Neuropsychological deficits and morphological MRI brain scan abnormalities in apparently healthy non-encephalopathic patients with cirrhosis A controlled study. J Hepatol. 1989;9:319-25.

19. Norenberg MD. Astrocytic-ammonia interactions in hepatic encephalopathy. Semin Liver Dis. 1996; 16:245-52.

20. O'Donnell BF, Squires NK, Martz MJ, Chen JR, Phay AJ. Evoked potential changes in neuropsychological performance in Parkinson's disease. Biol Psychol. 1987;24:23-7.

21. O'Donnell BF, Friedman S, Swearer JM, Drachman DA. Active and passive P3 latency and psychometric performance: influence of age and individual differences. Int J Psychophysiol. 1992;12):187-95.

22. Ortiz M, Jacas C, Cordoba J. Minimal hepatic encephalopathy: diagnosis, clinical significance and recommendations. J Hepatol. 2005;42(Suppl 1):s45-s53.

23. Pappas SC, Jones EA. Methods for assessing hepatic encephalopathy. Semin Liver Dis. 1983;3:298-307.

24. Polich J. P300 in clinical applications: meaning, method, and measurement in electroencephalography: basic principles, clinical applications, and related fields, 3rd ed. Baltimore: Williams \& Wilkins: 1993. p.1005.

25 .Pugh RN, Murray-Lion IM, Dawson JL, Pietroni MC, Williams R. Transection of the oesophagus for bleeding oesophageal varices. Br J Surg. 1973;60:646-9.

26. Quero JC, Shalm SW. Subclinical hepatic encephalopathy. Semin Liver Dis. 1996; 16:321-8

27. Romero-Gómez M, Boza F, García-Valdecasas MS, García E, Aguilar-Reina J. Subclinical hepatic encephalopathy predicts the development of overt hepatic encephalopathy Am J Gastroenterol. 2001;96:2718-23.

28. Saxena N, Bhatia M, Joshi YK, Garg PK, Tandon R. Auditory P300 event-related potentials and number connection test for evaluation of subclinical hepatic encephalopathy in patients with cirrhosis of the liver: a follow-up study. J Gastroenterol Hepatol 2001;16:322-7.

29. Saxena N, Bhatia M, Joshi YK, Garg PK, Dwivedi SN, Tandon RK. Electrophysiological and neuropsychological tests for the diagnosis of subclinical hepatic encephalopathy and prediction of overt encephalopathy. Liver. 2002;22:190-7.

30. Schomerus H, Schreiegg J. Prevalence of latent portasystemic encephalopathy in an unselect population of patients with liver cirrhosis in general practice. Z Gastroenterol. 1993;31:231-4.

31. Stahl J. Studies of the blood ammonia in liver disease. Ann Intern Med. 1963;58: $1-24$.

32. Watanabe A, Sakai T, Sato S, Imai F, Otho M, Arakawa Y, Toda G, Kobayashi K, Muto Y, Tsujii T, Kawasaki H, Okita K, Tanikawa K, Fujiyama S, Shimada S. Clinical efficacy of lactulose in cirrhotic patients with and without subclinical hepatic encephalopathy. Hepatology. 1997;26:1410-4 
33. Wein C, Koch H, Popp B, Oehler G, Schauder P. Minimal hepatic encephalopathy impairs fitness to drive. Hepatology. 2004;39:739-45.

34. Weissenborn K, Scholz M, Hinrichs H, Wiltfang J, Schmidt FW, Kunkel H. Neurophysiological assessment of early hepatic encephalopathy. Electroenceph Clin Neurophysiol. 1990;75:289-95.

35. Weissenborn K, Ruckert N, Hecker H, Manns MP. The number connection tests A and $\mathrm{B}$ : interindividual variability and use for the assessment of early hepatic encephalopathy. J Hepatol. 1998;28:646-53.

36. Weissenborn K, Heidenreich S, Ennen J, Ruckert N, Hecker H. Attention deficits in minimal hepatic encephalopathy. Metab Brain Dis. 2001;16:13-9.
37. Yang SS, Wu CH, Chiang TR, Chen DS. Somatosensory evoked potentials in subclinical portosystemic encephalopathy: a comparison with psychometric tests. Hepatology. 1998;27:357-61.

38. Zeneroli ML, Cioni G, Ventura P, Russo AM, Venturini I, Casalgrandi G, Ventura E. Interindividual variability of the number connection test. J Hepatol. 1992;15:263-4.

Recebido em 29/5/2006 Reapresentado em 12/11/2008.

Aprovado em 24/12/2008 